\title{
Perfil demográfico e socioeconômico de manipuladores em uma unidade de alimentação e nutrição na cidade de Juiz de Fora- MG
}

\author{
Demographic and Socioeconomic Profile of Manipulators \\ in a Food and Nutrition Unit
}

Daniel Inácio Peixoto Domingues de Azevedo', Rebeca Galhardo Tiburcio', Bruna Laiza Silva Marinho', Mirella Lima Binoti²

'Nutricionista, formado pela Universidade Federal de Juiz de Fora (UFJF)

${ }^{2}$ Nutricionista, Professora Associada, Universidade Federal de Viçosa (UFV)

E-mail para contato: Mirella Lima Binoti - mirella.binoti@ufv.br

\section{Resumo}

Mudanças decorrentes do processo de industrialização afetaram os costumes e o modo de se alimentar da sociedade, levando ao aumento do número de refeições realizadas fora do ambiente domiciliar bem como na quantidade de estabelecimentos produtores de alimentos, e para que isso seja possível, é essencial a presença dos manipuladores de alimentos. O objetivo deste trabalho foi descrever o perfil demográfico e sócio econômico dos manipuladores de alimentos de uma Unidade de Alimentação e Nutrição. Trata-se de estudo transversal realizado na matriz de uma Unidade de Alimentação e Nutrição (UAN), na cidade de Juiz de Fora, Brasil. Foi aplicado um questionário autoadministrado contendo informações demográficas e socioeconômicas. Os dados foram descritos por meio da distribuição de frequências absolutas e relativas. Participaram da pesquisa 81 manipuladores de alimentos, agrupados de acordo com a função que exercem na unidade. Os participantes apresentam idade média de 33,9 anos $( \pm 9,5$ anos), a maioria pertence ao gênero masculino $(61,7 \%)$ e reside na cidade de Juiz de Fora (97,5\%), 84\% apresenta o ensino fundamental e ensino médio; 48,1\% é de auxiliar de cozinha; o tempo de profissão $(72,8 \%)$ e tempo exercido de profissão na empresa $(55,6 \%)$ apresentam período superior a um ano. A implementação constante e periódica de treinamentos, e incentivo à capacitação, são importantes. Além disso, deve-se considerar um gerenciamento de recursos humanos que promova a qualidade de vida e a valorização do indivíduo no ambiente organizacional.

Palavras-chave: Alimentos. Capacitação. Trabalhadores.

\begin{abstract}
Changes resulting from the industrialization process affected society's customs and way of eating, leading to an increase in the number of meals taken outside the home environment as well as in the number of food producing establishments, and for this to be possible, it is essential to presence of food handlers. The objective of this work was to describe the demographic and socioeconomic profile of food handlers in a Food and Nutrition Unit. This is a crosssectional study conducted at the headquarters of a Food and Nutrition Unit, in
\end{abstract}


Juiz de Fora, Brazil, a self-administered questionnaire containing demographic and socioeconomic information was applied. The data were described through the distribution of absolute and relative frequencies. 81 food handlers participated in the research, grouped according to their role in the unit. The participants have an average age of 33.9 years ( \pm 9.5 years), most of them are male (61.7\%) and live in the city of Juiz de Fora (97.5\%), 84\% have the elementary school to high school; 48.1\% are kitchen assistants; time in the profession (72.8\%) and time in the company (55.6\%) is longer than one year. In view of the low level of education observed and the short time spent in the function, human resources management that promotes quality of life and the valuation of the individual in the organizational environment should be considered. In addition, constant and periodic implementation of training and incentives for capacity are important.

Keywords: Food. Training. Workers.

\section{INTRODUÇÃO}

A alimentação constitui uma das principais atividades exercidas pelo homem. Podese dizer que o ato de comer possui característica multidimensional e um significado muito mais amplo que o da simples garantia da sobrevivência. Desta forma, a alimentação se relaciona com fatores biológicos, sociais, culturais, diretamente envolvidos na transformação da matéria prima, até o consumo final ${ }^{1}$.

No Brasil, a partir da segunda metade do século XX, ocorreram mudanças decorrentes do processo de industrialização que afetaram os costumes e o modo de se alimentar da sociedade. Pode se dizer que, deste período em diante, houve aumento significativo do número de refeições realizadas fora do ambiente domiciliar bem como na quantidade de estabelecimentos produtores de alimentos ${ }^{1}$.

Mediante estas transformações, o hábito de comer fora de casa se tornou quase que uma "imposição" em função dos longos deslocamentos e da extensiva jornada de trabalho enfrentada por uma significativa parcela da população². Desta maneira, a alimentação realizada em Unidades de Alimentação e Nutrição (UAN), tornou-se uma realidade em um mercado que vem se expandindo cada vez mais em países como Brasil, EUA e também na Europa 3 .

Para que essa alimentação fora do lar seja possível, é essencial a presença dos indivíduos que executam as atividades nas UANs, também denominados manipuladores de alimentos, que segundo a RDC 216/2004, são classificados como "qualquer pessoa do serviço de alimentação que entra em contato direto e indireto com o alimento"4. 
Os manipuladores de alimentos apresentam grande importância para a produção de alimentos com qualidade higiênico-sanitária, e nesse contexto, a segurança do trabalho e a utilização de EPIs dentro de uma Unidade de Alimentação e Nutrição devem ser garantidas para que haja a proteção de cada manipulador e da equipe. Os manipuladores de alimentos na Unidade de Alimentação estudada são conscientes sobre a importância da utilização dos EPIs. Entretanto, percebe-se que há defasagem em relação a sua utilização e que a maioria dos colaboradores não sabe quando e como utilizar cada equipamento 5 .

Diante disso, pretende-se com a realização deste estudo descrever o perfil demográfico e socioeconômico dos manipuladores de uma Unidade de Alimentação e Nutrição localizada na cidade de Juiz de Fora - MG.

\section{MATERIAIS E MÉTODOS}

Estudo transversal realizado na matriz de uma Unidade de Alimentação e Nutrição (UAN), na cidade de Juiz de Fora - MG, Brasil. A unidade possui 120 manipuladores de alimentos, produz 10000 refeições por dia, oferece almoço em dois locais e distribui refeições transportadas para várias unidades da cidade, atuando nos ramos de refeições hospitalares, prisionais, escolares e empresariais.

A coleta de dados foi realizada no período de 05 de outubro a 05 de novembro de 2017, por estudantes de nutrição com o auxílio da nutricionista responsável pelo local e sob supervisão da professora orientadora, por meio de um questionário auto administrado (o próprio participante preenche as respostas). Os colaboradores foram convidados a participar, aleatoriamente, no intervalo de alimentação ou descanso, sendo esclarecidos os objetivos e o modo de realização do estudo. Com o objetivo de aumentar a validade das respostas, esclareceu-se ao participante que o questionário não fazia parte da avaliação individual de desempenho.

Os manipuladores que concordaram em participar assinaram o termo de consentimento livre esclarecido. No desenvolvimento do Projeto foram considerados os princípios éticos que norteiam a Pesquisa em Seres Humanos, contidos na Resolução CNS 466/12 com a Norma Operacional No 001/2013 CNS. O projeto foi submetido ao Comitê de Ética em Pesquisa da Universidade Federal de Juiz de Fora e foi executado mediante aprovação (Número do Parecer: 2.147.068).

Foram incluídos todos os manipuladores de alimentos que aceitaram participar, não estavam em férias ou licença-saúde no período da pesquisa, idade acima de 18 anos, 
ambos os gêneros, e que sabiam ler e escrever. Foram excluídos os colaboradores que, nos dias programados para coleta de dados, não estiveram disponíveis para participar e/ou estiveram impossibilitados em responder ao questionário por apresentarem alguma limitação funcional e/ou motora.

O instrumento utilizado para coleta de dados foi dividido em duas partes. A primeira delas contendo variáveis de informações demográficas: gênero, com as opções para masculino e feminino; estado civil (com as opções: casado, solteiro e separado/divorciado/viúvo); idade e se possui residência fixa. Na segunda parte, constavam as variáveis de informações socioeconômicas com dados a serem preenchidos sobre níveis de escolaridade e também as seguintes perguntas sobre a profissão exercida: "qual a função exerce na empresa?"; "quanto tempo exerce essa profissão no mercado de trabalho?"; "Quanto tempo exerce essa profissão na empresa?".

A população-alvo da pesquisa foram os colaboradores operacionais e técnicos da unidade. Para o cálculo da amostra foi utilizada a fórmula:

$$
\mathrm{n}=\left[\operatorname{EDFF}^{*} \mathrm{~Np}(1-\mathrm{p})\right] /\left[\left(\mathrm{d} 2 / \mathrm{Z} 21-\alpha / 2 *(\mathrm{~N}-1)+\mathrm{p}^{*}(1-\mathrm{p})\right]\right.
$$

Onde,

- Tamanho da população (para o fator de correção da população finita ou fcp) $(\mathrm{N}): 120$

- Frequência hipotética da população (p): 50\%

- Limites de confiança (d): $5 \%$

- Efeito do desenho (EDFF): 1

- Escore padrão da distribuição normal (Z): (1,96 para 95\% de confiança)

Conforme resultado do OpenEpi, Versão 3, calculadora de código aberto-SSPropo a amostra não probabilística por demanda foi de 93 manipuladores de alimentos, diretos e indiretos.

As informações obtidas foram armazenadas em um banco de dados elaborado para este fim em microcomputador, com o auxílio do aplicativo Excel 11.o. Os dados foram descritos por meio da distribuição de frequências absolutas e relativas, processados 
Daniel Inácio Peixoto Domingues de Azevedo, Rebeca Galhardo Tiburcio, Bruna Laiza Silva Marinho, Mirella Lima Binoti | Perfil demográfico e socioeconômico de manipuladores em uma unidade de alimentação e nutrição na cidade de Juiz de Fora-MG

e analisados por meio do programa Statistical Software for Professionals (STATA), versão 13.0. A análise de frequência foi realizada para variáveis categóricas.

\section{RESULTADOS}

O presente trabalho contou com a participação de 97 colaboradores, tanto manipuladores diretos, quanto indiretos de alimentos. No entanto, apenas 81 questionários foram analisados, devido à algumas respostas incompletas.

Em relação à variável idade, observou-se que os participantes apresentam idade média de 33,9 anos ( $\pm 9,5$ anos).

As informações demográficas e socioeconômicas podem ser visualizadas na Tabela 1. Para a variável gênero, observou-se que $62,9 \%(n=51)$ dos participantes são homens e $37,1 \%(n=30)$ são mulheres. Quanto ao estado civil, observou-se que 48,1\% (n=39) dos participantes eram solteiros, 45,7\% (n=37) casados e 6,2\% (n=5) divorciados ou viúvos.

Tabela 1 - Dados demográficos dos manipuladores de uma unidade de alimentação e nutrição de Juiz de Fora - MG, 2017.

\begin{tabular}{llll}
\hline & & $\begin{array}{c}\text { Porcentagem } \\
\text { (\%) }\end{array}$ & $\begin{array}{c}\text { Total } \\
\text { (n) }\end{array}$ \\
\hline $\begin{array}{l}\text { Total de } \\
\text { Entrevistados }\end{array}$ & & $100 \%$ & 81 \\
\hline Gênero & Masculino & $62,9 \%$ & 51 \\
\hline & Feminino & $37,1 \%$ & 30 \\
\hline Estado Civil & Solteiro & $48,1 \%$ & 39 \\
\hline & Casado & $45,7 \%$ & 37 \\
\hline Local onde residem & Divorciados ou viúvos & $6,2 \%$ & 5 \\
\hline & Juiz de Fora & $97,5 \%$ & 79 \\
\hline & Outros & $2,5 \%$ & 2 \\
\hline & Até Ensino Fundamental* & $24,7 \%$ & 20 \\
\hline & Ensino Médio & $59,3 \%$ & 48 \\
\hline
\end{tabular}

*Inclui analfabetos e aqueles que declaram saber ler e escrever

No que se refere à escolaridade, verificou-se que $24,7 \%(\mathrm{n}=20)$ dos colaboradores possuem até ensino fundamental, 59,3\% $(n=48)$ possuem o ensino médio e $16,0 \%$ ( $n=13)$ formação superior. 
Em relação à função exercida, verificou-se a seguinte distribuição percentual dos colaboradores: 8,6\% $(n=7)$ líderes de setor e nutricionistas; 6,2\% $(n=5)$ cozinheiros; 3,7\% $(n=3)$ trabalham na expedição e 3,7\% $(n=3)$ na câmara fria; 13,6\% $(n=11)$ são estoquistas; $11,1 \%(n=9)$ atuam no setor de pré-preparo; 5,0\% $(n=4)$ são açougueiros e 48,1\% (n=39) são auxiliares de cozinha.

Em relação ao tempo exercido de profissão (Figura 1), pode-se observar que 27,2\% $(\mathrm{n}=22)$ mantiveram-se no mesmo cargo por um intervalo de tempo inferior a um ano e $72,8 \%(n=59)$ dos entrevistados exerciam a mesma função por um período maior que um ano.

Figura 1 - Distribuição por tempo exercido de profissão, dos manipuladores de uma unidade de alimentação e nutrição de Juiz de Fora - MG

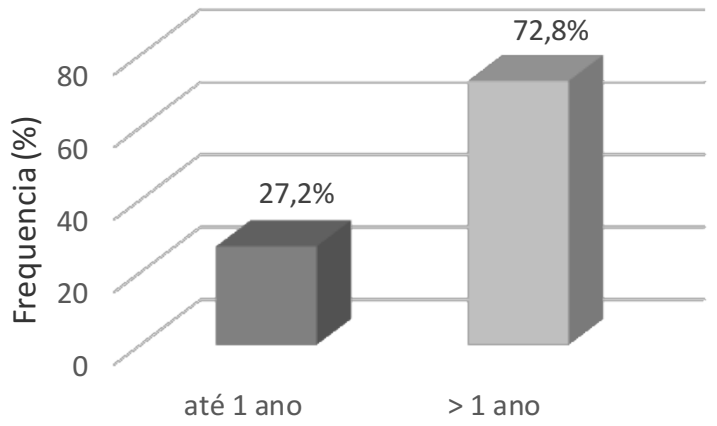

Sobre o tempo exercido de profissão na empresa (Figura 2), verificou-se que $44,4 \%$ $(n=36)$ estão a menos de um ano no local, enquanto 55,6\% (n=45) dos colaboradores apresentam período superior a um ano de atividade na empresa.

Figura 2 - Distribuição por tempo exercido na função na empresa, dos manipuladores de uma unidade de alimentação e nutrição de Juiz de Fora - MG.

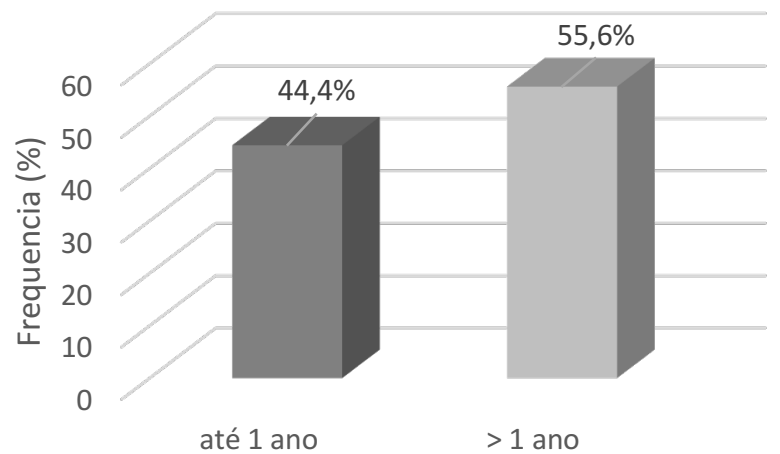




\section{DISCUSSÃO}

As cozinhas são caracterizadas por trabalho intenso, onde é exigido alta produtividade em um curto período, com atividades manuais intensas e automatizadas, aliadas, muitas vezes, às infraestruturas impróprias. Condições inadequadas de trabalho, com problemas no ambiente, nos equipamentos e máquinas, acabam levando a insatisfações, cansaço excessivo, quedas de produtividade, problemas de saúde e acidentes de trabalho ${ }^{6}$.

Sobre a idade dos manipuladores nessa pesquisa, foi verificada uma idade média de aproximadamente 40 anos. Faixa etária semelhante a observada por Arantes et al. ${ }^{7}$ e por Sousa et al. ${ }^{8}$ Segundo Byrd-Bredbenner ${ }^{9}$ determinados grupos de indivíduos, como jovens adultos são mais propensos a se envolverem em situações de risco. Embora ainda não estejam claros os motivos que favoreçam a este quadro, acreditase que a falta de oportunidades e de educação sobre a manipulação correta dos alimentos possam influenciar o comportamento deste grupo ${ }^{9}$.

A presença de indivíduos com faixas etárias menores na área de manipulação de alimentos é corriqueira. Esse quadro demonstra a necessidade de capacitação profissional, uma vez que a maioria desses indivíduos se encontra na primeira experiência de trabalho, necessitando, portanto, de conhecimentos teóricos para realização das atividades a serem desenvolvidas ${ }^{8}$.

O serviço realizado em uma UAN é predominantemente feito por homens ${ }^{10}$. No presente trabalho foi observado que a maioria dos manipuladores de alimentos era do gênero masculino. Assim como verificado por Arantes et al.7 em estudo recente conduzido em uma UAN de um campus pertencente a um Instituto Federal localizado em Minas Gerais, onde verificou-se que $61,5 \%$ dos manipuladores também eram representados por esse gênero, e por Barreto et al. ${ }^{10}$ No entanto, a predominância feminina de manipuladores na área de alimentação, foi encontrada por outros autores $^{8,11}$. O aumento da participação da mulher no mercado de trabalho é um fenômeno progressivo e passou a ocorrer com maior intensidade a partir da Segunda Guerra Mundial, concomitante ao processo de industrialização, onde a população passou a realizar cada vez mais as refeições fora de casa, principalmente por fatores como a atuação da mulher no mercado de trabalho e a intensificação populacional nos grandes centros, estimulando um aumento expressivo em estabelecimentos de comercialização e produção de alimentos ${ }^{12}$. 
Sobre o estado civil dos manipuladores de alimentos, foram considerados aqueles que eram legalmente casados juntamente com o grupo de pessoas que viviam sob o regime de união estável, foi observado nesse estudo, predominância de manipuladores solteiros, diferente de outras pesquisas, em que foi constado uma maioria dos participantes representada por indivíduos que residiam com seus parceiros $^{8,11}$.

Com o intuito de compreender os diversos fatores que determinam o comprometimento do indivíduo em relação à organização e a profissão, Bastos et al. ${ }^{12}$ investigaram 1678 trabalhadores em um estudo que envolveu 42 empresas, de médio e grande porte do setor industrial. Verificou-se que indivíduos casados apresentaram níveis mais elevados de comprometimento organizacional e com a profissão sendo assim, classificados pelo estudo na categoria denominada duplo comprometimento. Estes, também apresentaram maior percentual na categoria comprometimento unilateral com a organização, que representa um compromisso mais significativo com a empresa do que com a ocupação exercida. Por sua vez, os indivíduos solteiros situaram-se em maior proporção, na categoria duplo descompromisso, que é aquela onde ocorre menor comprometimento com a organização e com a profissão ${ }^{12}$.

Nesse estudo foi verificada uma escolaridade acima da média, visto que mais da metade dos manipuladores relataram possuir o ensino médio. Semelhante ao observado por Sousa et al. ${ }^{8}$ e Oliveira et al. ${ }^{11} \mathrm{O}$ grau de escolaridade constatado pode ser visto como aspecto positivo, pois indivíduos com baixa escolaridade têm mais dificuldade, são mais lentos e cometem mais erros relacionados à execução do trabalho ${ }^{8}$.

O nível de escolaridade influencia na capacidade de aprendizado de manipuladores de alimentos e torna-se uma dificuldade em cursos de capacitação que devem ser realizados dentro de uma UAN. Manipuladores de alimentos que concluem o $2^{\circ} \mathrm{grau}$ ou que possuem curso superior apresentam uma melhor compreensão sobre as condições de higiene pessoal do que aqueles com menor nível de educacional ${ }^{13}$. Além disso, a baixa escolaridade pode contribuir diretamente para a ocorrência de acidentes ${ }^{14}$.

A minoria dos manipuladores possuía formação superior. É importante desatacar que o processo educativo, ou seja, pessoas com formação específica, pode ser facilitador de melhores condições de saúde, conforme observado em estudo que 
discute a educação em saúde na melhora da qualidade de vida no trabalho e queixas álgicas ocupacionais em manipuladores de alimentos em ambiente hospitalar ${ }^{11}$.

Estudos mostram que a ergonomia está diretamente relacionada com a qualidade do trabalho realizado e que os problemas ergonômicos associados à sobrecarga das atividades podem comprometer a saúde dos trabalhadores ${ }^{14}$. Os principais problemas vivenciados pelos manipuladores de alimentos são caracterizados por trabalho intenso, onde é exigido alta produtividade em um curto período, com atividades intensas manuais e automatizadas, aliadas, muitas vezes, às infraestruturas impróprias. Além de condições inadequadas de trabalho, com problemas no ambiente, nos equipamentos e máquinas acabam levando a insatisfações, cansaço excessivo, quedas de produtividade, problemas de saúde e acidentes de trabalho ${ }^{6}$. Fatores que interferem na qualidade do serviço.

Ao analisar a escolaridade pode-se vincular às funções exercidas na empresa, onde verificou-se a maioria dos cargos exercidos não necessitam de uma formação específica.

Nessa pesquisa foi verificado que os manipuladores em sua maioria, permaneciam por mais de um ano na mesma profissão e na mesma função na empresa estudada. Assim como observado por Sousa et $\mathrm{al}^{8}$. A influência do tempo no exercício do cargo pode interferir na percepção do manipulador de alimentos sobre a higiene alimentar, uma vez que o maior ou menor tempo dedicado à mesma função contribui, de alguma forma, nos conhecimentos adquiridos ${ }^{15}$.

Na cidade de Porto, em Portugal, a influência positiva sobre o tempo de permanência na profissão em relação ao conhecimento dos participantes em questões sobre higiene e segurança alimentar foi verificada. A experiência profissional demonstrou relevância considerável em relação ao nível de conhecimento adquirido, de acordo com a experiência na mesma função ${ }^{16}$. Entretanto, a experiência profissional, de forma geral, é considerada um fator importante para a promoção da qualidade do trabalho, uma vez que repercute positivamente sobre a produtividade do trabalhador. Desta forma, profissionais que mantêm maior constância em uma mesma função, via de regra, são mais produtivos em relação aos que trocam de ocupação ou conquistam novos cargos ${ }^{13}$. O maior tempo de trabalho numa mesma empresa propicia um maior controle e compreensão dos procedimentos aplicados no trabalho ${ }^{17}$. 
Outra questão a ser considerada e que se relaciona com a permanência na empresa diz respeito à satisfação geral do trabalhador. A satisfação geral no trabalho está intimamente relacionada ao bem-estar individual. Neste contexto, é considerada uma questão de grande importância tanto para trabalhadores quanto por parte das empresas, podendo afetar o comportamento do indivíduo, a produtividade e indicar as intenções dos trabalhadores em abandonarem o emprego ${ }^{17}$.

\section{CONCLUSÃO}

As informações obtidas pelo estudo evidenciaram a presença de uma força de trabalho predominante marcada por um grau de escolaridade médio, representada por jovens adultos e indivíduos solteiros. Com relação ao tempo exercido de profissão verificou-se que mais da metade dos participantes da pesquisa apresenta tempo de permanência na empresa por um período superior a um ano.

Frente aos resultados apresentados, foi possível a caracterização do perfil demográfico e socioeconômico de manipuladores de alimentos. Esses dados possibilitarão desenhos metodológicos específicos a serem utilizados em capacitações com os mesmos, aprimorando o conhecimento, a segurança e a capacidade técnica desses indivíduos, garantindo assim a produção de alimentos seguros e de qualidade, visando a qualidade higiênico-sanitária dos alimentos.

Além disso, deve-se considerar um gerenciamento de recursos humanos que promova a qualidade de vida e a valorização do indivíduo no ambiente organizacional.

\section{Agradecimento}

Universidade Federal de Juiz de Fora pela bolsa de iniciação cientifica.

\section{REFERÊNCIAS}

1. Monteiro MAM. A importância da ergonomia na saúde dos funcionários de Unidades de Alimentação e Nutrição. Revista Baiana de Saúde Pública. 2012; 33 (3): 416-427.

2. Albuquerque EM, Seabra LMAJ, Rolim PM, Gomes LM. Riscos físicos em uma Unidade de Alimentação e Nutrição: implicações na saúde do trabalhador. Revista Associação Brasileira de Nutrição. 2012; 4(5).

3. Akutsu RM, Botelho RA, Camargo EB, Sávio KEO, Araújo WC. Adequação das boas práticas de fabricação em serviços de alimentação. Revista de Nutrição. 2005; 18(3). 
Daniel Inácio Peixoto Domingues de Azevedo, Rebeca Galhardo Tiburcio, Bruna Laiza Silva Marinho, Mirella Lima Binoti | Perfil demográfico e socioeconômico de manipuladores em uma unidade de alimentação e nutrição na cidade de Juiz de Fora-MG

4. Brasil, Agência Nacional de Vigilância Sanitária. Resolução RDC nº 216, de 15 de setembro de 2004 .

5. Tiburcio RG, Azevedo DIPD, Marinho BLS, Binoti ML. Personal protective equipment use by food manipulators in a food and nutrition unit. HU Revista. 2020; 45(1):13-21.

6. Velasco JC, Molina VBC. Condições de trabalho, saúde e segurança dos colaboradores das unidades de alimentação e nutrição. Revista Multidisciplinar da Saúde (RMS). 2020; 2(03): 16-31.

7. Arantes RS, Benevenuto WCAN, Benevenuto Júnior AA, Martins ADO, Martins EMF, Cruz WF. Características sociodemográficas e conhecimentos dos manipuladores de alimentos sobre as Boas Práticas, antes e após treinamento, em uma Unidade de Alimentação e Nutrição. Alimentos: Ciência, Tecnologia e Meio Ambiente. 2020; 1(7): 108-125.

8. Sousa APM, Souza FLopes, Duarte ECPS, Cabral SMR, Santos MM. Socioeconomic profile of food handlers of the municipal education district of a city in the state of Piauí. Brazilian Applied Science Review. 2019, 3(2): 908-917.

9. Byrd-Bredbenner et al. Food Safety Self-Reported Behaviors and Cognitions of Young Adults: Results of a National Study. Jornal of Food Protection. 2007; 70(8) 1917:1926.

10. Barreto LKS, Leone NMCPG, Nascimento LC, Aouar WAE. Trabalho, prazer e sofrimento: um estudo em Unidades de Alimentação e Nutrição. Razon y Palabra. 2016; 20 (3).

11. Oliveira SP de FP, Ferreira AMM, Teixeira GM. Qualidade de vida no trabalho de manipuladores de um serviço de nutrição hospitalar em Maceió - AL. Revista Eletrônica Acervo Saúde. 2020; (50):e3498.

12. Bastos AVB, Correia NCN, Lira SB. Padrões de comprometimento com a profissão e a organização: o impacto de fatores pessoais e da natureza do trabalho. Revista de Administração da Universidade de São Paulo. 2000; 35(4).

13. BRASIL, Secretaria de Assuntos Estratégicos. Determinantes da produtividade do trabalho para a estratégia da sustentabilidade e promoção da classe média, Brasília, 2013.

14. Dias GN, Aires IO, Sousa FRO de, Araújo MC, Moura ACC, Lima SMT, Moura Menêzes JV, da Silva MS, Revoredo CMS. A importância da ergonomia em unidades de alimentação e nutrição: Uma revisão integrativa. Revista Eletrônica Acervo Saúde. 2020; 38: e1680

15. Viveiros FC. Avaliação de conhecimentos de higiene e segurança alimentar de manipuladores de alimentos em unidades de alimentação e nutrição do sector hospitalar. [Trabalho de investigação]. Porto: Universidade do Porto. Porto, 2010.

16. Góios A, Martins ML, Ferreira L, Rocha ANA. Conhecimentos de manipuladores de alimentos sobre segurança dos alimentos e alergias. Higiene Alimentar. 2017;31(264/265): 38-44.

17. Nunes HPG. Satisfação no trabalho dos manipuladores de alimentos. [Trabalho de investigação] Porto: Universidade do Porto. Porto, 2010.

Submissão: 07/04/2020

Aprovação: 05/01/2021 